\title{
A Multi-scale Finite Element Approach for the Random Mechanical Response of Honeycomb-cored Structures
}

\author{
E.I. Saavedra Flores ${ }^{1,2, *}$, F.A. DiazDelaO ${ }^{1, *}$, M.I. Friswell ${ }^{1, \dagger}$ and J. Sienz ${ }^{1, \dagger}$ \\ ${ }^{1}$ College of Engineering, Swansea University, Singleton Park, Swansea SA2 8PP, United Kingdom \\ ${ }^{2}$ Departamento de Ingeniería en Obras Civiles, Universidad de Santiago de Chile, \\ Av. Ecuador 3659, Santiago, Chile
}

\begin{abstract}
This paper studies the uncertainty in the mechanical response of foam-filled honeycomb cores by means of a computational multi-scale approach. A finite element framework is adopted to determine the response of a periodic arrangement of aluminum honeycomb core filled with PVC foam. By considering uncertainty in the geometric properties of the microstructure, a significant computational cost is added to the solution of a large set of microscopic equilibrium problems. In order to tackle this high cost, we combine two strategies. Firstly, we make use of symmetry conditions present in a representative volume element of material. Secondly, we build a statistical approximation to the output of the computer model, known as a Gaussian process emulator. Following this double approach, we are able to reduce the cost of performing uncertainty analysis of the mechanical response. In particular, we are able to estimate the 5-th, 50-th, and 95-th percentile of the mechanical response without resorting to more computationally expensive methods such as Monte Carlo simulation.
\end{abstract}

\section{Nomenclature}

$C(\cdot, \cdot)$ covariance structure

$G \quad$ virtual work functional

$m^{* *}(\cdot)$ predictive mean

$\boldsymbol{u}_{\mu} \quad$ local displacement field

$\tilde{\boldsymbol{u}}_{\mu} \quad$ displacement fluctuation field

$\boldsymbol{x} \quad$ arbitrary point of the macroscopic continuum

$\boldsymbol{y}$ arbitrary point of the microscopic domain

$\varepsilon \quad$ macroscopic or homogenised strain tensor

$\varepsilon_{\mu} \quad$ microscopic strain tensor field

$\eta(\cdot) \quad$ emulator

$\boldsymbol{\sigma}$ macroscopic or homogenised stress tensor

$\sigma_{\mu} \quad$ microscopic stress tensor

$\Omega_{\mu} \quad$ Microscopic domain

\section{Introduction}

Over the last two decades, homogenisation-based multi-scale constitutive modelling techniques relying on the volume averaging of the stress and strain fields over a Representative Volume Element (RVE) of material have attracted considerable attention within the computational mechanics community ${ }^{1-4}$. The interest in this area stems mainly from the suitability of multi-scale models of this type for finite element implementation

\footnotetext{
*Post-doctorate researcher, College of Engineering.

${ }^{\dagger}$ Professor, College of Engineering.
} 
and, probably more importantly, from their potential ability to capture the non-linear response by means of conventional internal variable-based phenomenological models. Such models offer the possibility of describing more accurately the stress response under complex strain paths, although they suffer from the drawback of excessive computing costs. These costs are usually acceptable when finite element analyses of single RVEs are carried out in the context of deterministic problems (refer, for instance, to Refs. 5, 3,6). However, when parametric uncertainty is considered in the context of multi-scale problems, the computational cost of solving the computer model can quickly become prohibitive. In such cases, even with the use of simple phenomenological models and relatively coarse RVE meshes, computing costs may rise by several orders of magnitude when compared to deterministic single-scale analyses.

In recent years, a significant number of components in engineering structures incorporate honeycomb cores $^{7}$ because of their high bending stiffness, strength and light weight. Important enhancements have been made in this context by filling the honeycomb system with polymer foams ${ }^{8-10}$. As noted by Burlayenko and Sadowski ${ }^{11}$, this method is inexpensive and does not add a significant amount of weight to a sandwich structure. As a result, the honeycomb microstructure with incorporated foam is more effective to withstand crash, impact and fatigue loading conditions ${ }^{11}$. Nevertheless, the mechanical properties of polymers show a significant variability due to porosity, microscopic cracks and inclusions. Furthermore, the honeycomb-cored material systems present considerable scatter because of manufacturing induced-defects, such as misalignment and asymmetries in the metallic components. Because of its increasing importance in engineering applications, this paper investigates the uncertainty in the mechanical response of foam-filled honeycomb cores by means of a computational multi-scale approach.

In order to address the issue of the very high computational cost introduced by considering parametric uncertainty in a fully coupled multi-scale analyses, we make use of implicit symmetry conditions present in the RVE of a material. Here, staggered-translational and point symmetry ${ }^{12,13}$ are considered at the same time, which leads to the choice of the smallest possible RVE domain. In addition, we employ a less expensive surrogate of a finite element model. This finite element model or code (henceforth referred to as a simulator) can be understood as a function $\eta: \mathbb{R}^{d_{1}} \rightarrow \mathbb{R}^{d_{2}}$ that, given an input $\mathbf{x}$, returns an output $\mathbf{y}=\eta(\mathbf{x})$. Several strategies that reduce the computational cost of expensive simulators by approximating their output can be found in the literature ${ }^{14,15}$. Based on different underlying methodologies, these strategies are referred to as metamodels, response surfaces, auxiliary models, among many others ${ }^{16}$. A particular kind of metamodel, usually employed in the analysis and design of computer experiments ${ }^{17,18}$, is Gaussian process emulation. This metamodel constructs a statistical approximation to the code's output, called a Gaussian process emulator (GPE). The main idea is the following: A small set of simulator runs is treated as training data used to update the prior beliefs about the output. As explained later, these prior beliefs take the form of a Gaussian stochastic process. After conditioning on the training data and updating, the mean of the resulting posterior distribution provides a fast approximation to the code's output at any untried input, whereas it returns the known value of the code at each of the initial runs. Gaussian process emulators have already been implemented in several scientific fields, such as test crash modelling ${ }^{19}$ and structural dynamics 20,21 .

\section{Multi-scale constitutive theory and numerical implementation}

The main assumption in the homogenisation-based multi-scale constitutive theories of solids is that the macroscopic or homogenised strain tensor $\boldsymbol{\varepsilon}$ at any arbitrary point $\boldsymbol{x}$ of the macroscopic continuum is the volume average of the microscopic strain tensor field $\varepsilon_{\mu}$ over the domain $\Omega_{\mu}$ of the local RVE. Similarly, the macroscopic or homogenised stress tensor field $\sigma$ is assumed to be the volume average of the microscopic stress tensor $\sigma_{\mu}$, over $\Omega_{\mu}$. For any instant $t$, the above conditions can be expressed mathematically as

$$
\varepsilon(\boldsymbol{x}, t)=\frac{1}{V_{\mu}} \int_{\Omega_{\mu}} \varepsilon_{\mu}(\boldsymbol{y}, t) d V \quad \text { and } \quad \boldsymbol{\sigma}(\boldsymbol{x}, t)=\frac{1}{V_{\mu}} \int_{\Omega_{\mu}} \boldsymbol{\sigma}_{\mu}(\boldsymbol{y}, t) d V,
$$

in which $V_{\mu}$ is the volume of the RVE associated to point $\boldsymbol{x}$, and $\boldsymbol{y}$ is the local RVE coordinates. Furthermore, the microscopic strain tensor $\varepsilon_{\mu}$ can be related to the local displacement field $\boldsymbol{u}_{\mu}$ by means of the standard expression $\varepsilon_{\mu} \equiv \nabla^{s} \boldsymbol{u}_{\mu}$, in which $\nabla^{s}$ is the symmetric gradient operator. In addition, it is possible to decompose the displacement field $\boldsymbol{u}_{\mu}$ as a sum of a linear displacement $\boldsymbol{\varepsilon}(\boldsymbol{x}, t) \boldsymbol{y}$, which represents a homogeneous strain, and a displacement fluctuation field $\tilde{\boldsymbol{u}}_{\mu}$. The displacement fluctuations field represents local variations about the linear displacement $\boldsymbol{\varepsilon}(\boldsymbol{x}, t) \boldsymbol{y}$ and do not contribute to the macroscopic scale strain. 
By taking into account the Hill-Mandel Principle of Macro-homogeneity ${ }^{22,23}$, which establishes that the macroscopic stress power must equal the volume average of the microscopic stress power over $\Omega_{\mu}$, the virtual work equation for the RVE can be reduced to

$$
\int_{\Omega_{\mu}} \boldsymbol{\sigma}_{\mu}(\boldsymbol{y}, t): \nabla^{s} \boldsymbol{\eta} d V=0
$$

with $\boldsymbol{\eta}$ representing the virtual kinematically admissible displacements field of the RVE.

In order to make problem (2) well-posed, a set of kinematical constraints upon the selected RVE is required. In what follows, the choice of this set will coincide with the widely used Periodic boundary displacement fluctuations model, which is typically associated with the modelling of periodic media. In this particular case, the RVE is a so-called unit cell whose periodic repetition generates the entire heterogeneous macro-continuum. Figure 1 shows a typical three-dimensional representation of a heterogeneous periodic

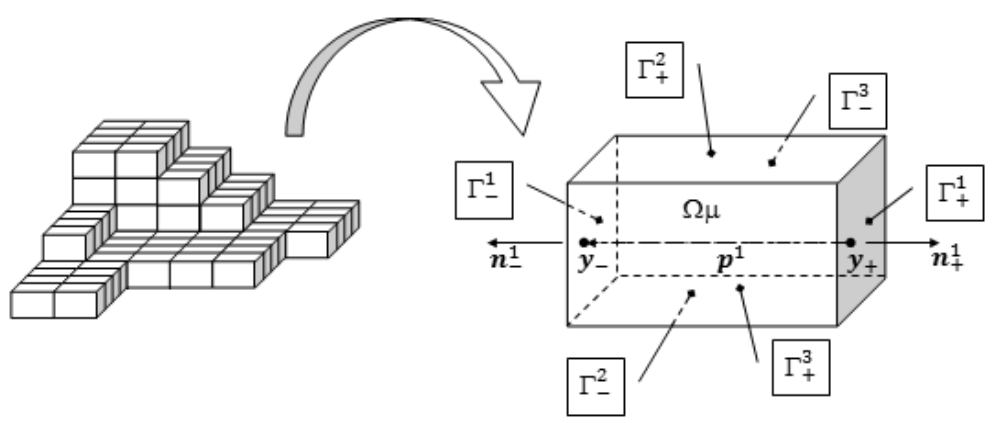

Figure 1. Three-dimensional periodic medium represented by parallelepiped-shaped unit cells

microstructure, formed by parallelepiped-shaped unit cells. In this case, each pair $j$ of cell sides consists of equally sized subsets $\Gamma_{+}^{j}$ and $\Gamma_{-}^{j}$ of $\partial \Omega_{\mu}$ with respective unit normal fields $\boldsymbol{n}_{+}^{j}$ and $\boldsymbol{n}_{-}^{j}$, such that $\boldsymbol{n}_{+}^{j}=-\boldsymbol{n}_{-}^{j}$ with a one-to-one correspondence between points $\boldsymbol{y}_{+}$and $\boldsymbol{y}_{-}$belonging respectively to the subsets $\Gamma_{+}^{j}$ and $\Gamma_{-}^{j}$. Here, the fundamental kinematical assumption consists of prescribing identical displacement fluctuation vectors for each pair of opposite points $\boldsymbol{y}_{+}$and $\boldsymbol{y}_{-}$on the RVE boundary $\partial \Omega_{\mu}$, such that:

$$
\tilde{\boldsymbol{u}}_{\mu}\left(\boldsymbol{y}_{+}, t\right)=\tilde{\boldsymbol{u}}_{\mu}\left(\boldsymbol{y}_{-}, t\right)
$$

By describing the RVE response by means of a generic local dissipative constitutive theory, the microscopic stress tensor $\sigma_{\mu}$ is represented by a functional of the history of $\varepsilon_{\mu}$, which is expressed symbolically as $\boldsymbol{\sigma}_{\mu}(\boldsymbol{y}, t)=\mathfrak{F}_{y}\left(\varepsilon_{\mu}^{t}(\boldsymbol{y})\right)$. The functional $\mathfrak{F}_{y}$ associated with point $\boldsymbol{y}$ maps the strain history, $\varepsilon_{\mu}^{t}$, up to time $t$, into the stress $\sigma_{\mu}$ of time $t$. In view of the above constitutive assumption and the additive decomposition of the microscopic displacement field, the equilibrium equation (2) leads to

$$
G\left(\varepsilon, \tilde{\boldsymbol{u}}_{\mu}, \boldsymbol{\eta}\right) \equiv \int_{\Omega_{\mu}} \mathfrak{F}_{y}\left\{\left[\varepsilon(\boldsymbol{x}, t)+\nabla^{s} \tilde{\boldsymbol{u}}_{\mu}(\boldsymbol{y}, t)\right]^{t}\right\}: \nabla^{s} \boldsymbol{\eta} d V=0
$$

where we have defined $G$ as virtual work functional.

In what follows, the numerical approximation of multi-scale constitutive models is described by means of the finite element method, when both staggered-translational and point symmetry ${ }^{12,13}$ are taken into account. A generic non-linear implicit finite element discretisation scheme is used as the underlying framework. The microscopic constitutive response that characterises the behaviour of the RVE material is assumed to be described within the framework of continuum thermodynamics with internal variables ${ }^{24,25}$.

The first crucial component of the implicit finite element approximation consists of an incremental (timediscrete) counterpart of the original microscopic constitutive law. In this case, an implicit numerical algorithm is used to discretise the rate constitutive equations of the internal variable-based model. Within a time interval $\Delta t=t_{n+1}-t_{n}$, with a given initial value $\boldsymbol{\alpha}_{n}$ for the set of internal variables at time $t_{n}$, the microscopic stress tensor $\left.\boldsymbol{\sigma}_{\mu}\right|_{n+1}$ at time $t_{n+1}$ is determined by the chosen numerical integration algorithm as 
a function of the microscopic strain tensor $\left.\varepsilon_{\mu}\right|_{n+1}$ at time $t_{n+1}$. This procedure gives rise to an approximate incremental constitutive function, $\hat{\boldsymbol{\sigma}}_{\mu}$, for the stress tensor, such that $\left.\boldsymbol{\sigma}_{\mu}\right|_{n+1}=\hat{\boldsymbol{\sigma}}_{\mu}\left(\varepsilon_{n+1}+\left.\nabla^{s} \tilde{\boldsymbol{u}}_{\mu}\right|_{n+1}, \Delta t ; \boldsymbol{\alpha}_{n}\right)$.

The following basic ingredient in the finite element approximation to the considered multi-scale models is the incremental counterpart of the microscopic equilibrium problem (4). By replacing the time-continuum constitutive functional $\mathfrak{F}_{y}$ of (4) with its time-discrete counterpart, $\hat{\boldsymbol{\sigma}}_{\mu}$, the incremental equilibrium problem of step $n+1$ can be obtained straightforwardly. Finally, to complete the numerical approximation of the model, a standard finite element discretisation $h$ is introduced. By replacing the domain $\Omega_{\mu}$ with its discrete counterpart $\Omega_{\mu}^{h}$, the fully spatial-temporal discretised version of (4) is obtained:

$$
G^{h}\left(\left.\tilde{\mathbf{u}}_{\mu}\right|_{n+1}\right) \equiv\left\{\int_{\Omega_{\mu}^{h}} \mathbf{B}^{\boldsymbol{\top}} \hat{\boldsymbol{\sigma}}_{\mu}\left(\boldsymbol{\varepsilon}_{n+1}+\left.\mathbf{B} \tilde{\mathbf{u}}_{\mu}\right|_{n+1}\right) d V\right\} \cdot \boldsymbol{\eta}=0
$$

in which $\mathbf{B}$ denotes the global strain-displacement matrix, $\boldsymbol{\varepsilon}_{n+1}$ is the prescribed finite element array of macroscopic engineering strains at time $t_{n+1}, \hat{\boldsymbol{\sigma}}_{\mu}$ is the the incremental constitutive functional at the RVE level that delivers the array of stress components, $\left.\tilde{\mathbf{u}}_{\mu}\right|_{n+1}$ is the array of global nodal displacement fluctuations and $\boldsymbol{\eta}$ is the array of global nodal virtual displacements.

Within the present framework, the algebraic equation system (5) is solved by the quadratically convergent Newton-Raphson iterative procedure. Then, with a given $\boldsymbol{\varepsilon}_{n+1}$, the following equation system is solved for the iterative correction $\delta \tilde{\mathbf{u}}_{\mu}^{(k)}$ to the displacement fluctuation during the typical Newton iteration $(k)$ :

$$
\left[\mathbf{F}^{(k-1)}+\mathbf{K}^{(k-1)} \delta \tilde{\mathbf{u}}_{\mu}^{(k)}\right] \cdot \boldsymbol{\eta}=0,
$$

where $\mathbf{F}^{(k-1)}$ is the residual vector, and $\mathbf{K}^{(k-1)}$ the tangent stiffness matrix.

For the specific purpose of this work, we exploit the existence of both staggered-translational and point symmetry conditions in a honeycomb configuration. In this context, the presence of these additional symmetries allows the choice of an RVE with domain comprising only one symmetric portion of the original domain. Whenever such conditions are met, substantial reductions in computational costs associated with CPU time and storage requirements are achieved.

In order to incorporate the above symmetry considerations in the finite element implementation of Equation (6), we reduce the analysis of the original full RVE domain into one quarter as shown in Figure 2, with the following subsets of nodes in the finite element mesh: one set of interior nodes, with corresponding

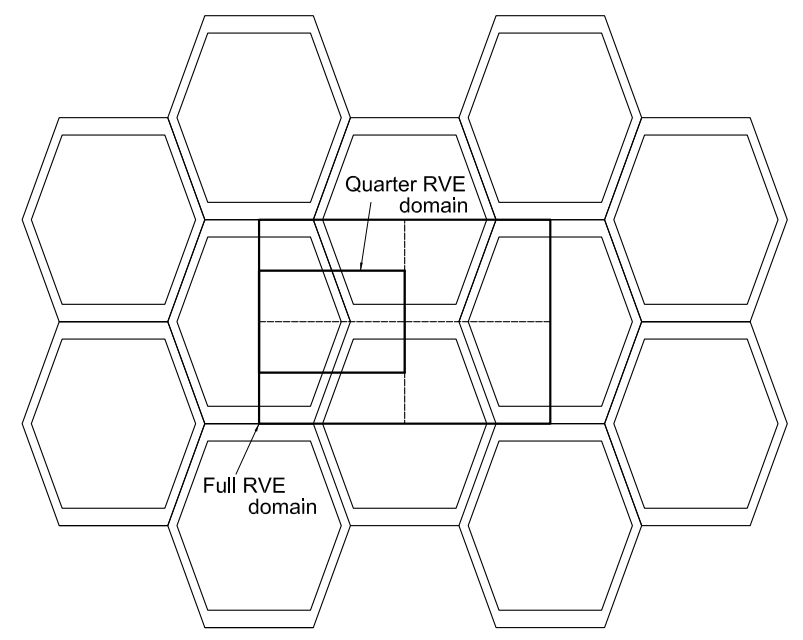

Figure 2. Reduction of the full RVE domain into one quarter.

quantities denoted by the subscript $i$; one set of corner nodes of the unit cell, denoted by $o_{1}, o_{2}, o_{3}$, and $o_{4}$; and two sets of boundary nodes denoted, respectively, with subscripts $\oplus$ and $\ominus$, so that for each node of set $\oplus$ with coordinate $\boldsymbol{y}_{\oplus}$ there is an equidistant node about the mid-point of each side with coordinate $\boldsymbol{y}_{\ominus}$ that belongs to the set $\ominus$ (refer to Figure 3 for further details). The relationship between $\oplus$ and $\ominus$ is such that a one-to-one correspondence is satisfied between these two sets of nodes. 


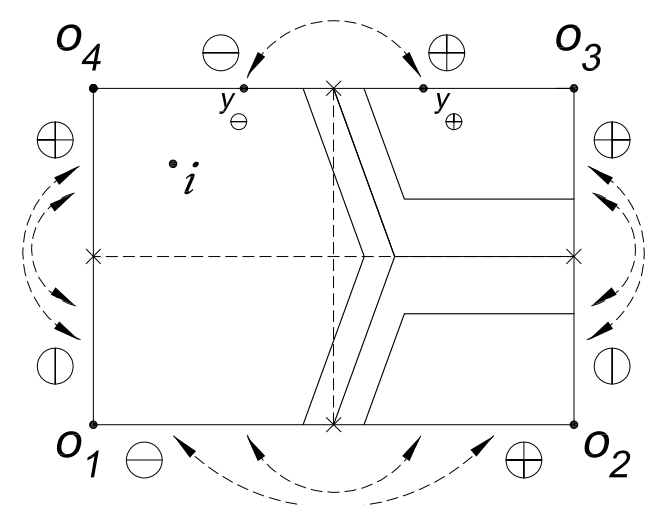

Figure 3. Schematic diagram of the quarter RVE domain chosen for the analyses. Set of points $i, o_{1} \ldots o_{4}, \oplus$ and $\ominus$ have been indicated here. The $\times$-symbols show the mid-point of each side of the reduced RVE domain such that equidistant points with coordinates $\boldsymbol{y}_{\oplus}$ and $\boldsymbol{y}_{\ominus}$ belonging to the sets $\oplus$ and $\ominus$, respectively, satisfy a one-to-one correspondence.

By applying this partition to the components of $\mathbf{F}, \mathbf{K}, \delta \tilde{\mathbf{u}}_{\mu}$ and $\boldsymbol{\eta}$, and by enforcing staggered-translational and point symmetry kinematical constraints simultaneously with Equation (3), the Newton-Raphson equation (6) can be explicitly written as ${ }^{12}$

$$
\begin{aligned}
& {\left[\begin{array}{cc}
\mathbf{k}_{i i} & \mathbf{k}_{i \oplus}-\mathbf{k}_{i \ominus} \\
\mathbf{k}_{\oplus i}-\mathbf{k}_{\ominus i} & \mathbf{k}_{\oplus \oplus}-\mathbf{k}_{\oplus \ominus}-\mathbf{k}_{\ominus \oplus}+\mathbf{k}_{\ominus \ominus} \\
\mathbf{k}_{o_{1} i}-\mathbf{k}_{O_{2} i}+\mathbf{k}_{o_{3} i}-\mathbf{k}_{o_{4} i} & \mathbf{k}_{o_{1} \oplus}-\mathbf{k}_{o_{1} \ominus}-\mathbf{k}_{O_{2} \oplus}+\mathbf{k}_{o_{2} \ominus}+\mathbf{k}_{o_{3} \oplus}-\mathbf{k}_{o_{3} \ominus}-\mathbf{k}_{o_{4} \oplus}+\mathbf{k}_{o_{4} \ominus}
\end{array}\right.} \\
& \left.\begin{array}{c}
\mathbf{k}_{i o_{1}}-\mathbf{k}_{i o_{2}}+\mathbf{k}_{i o_{3}}-\mathbf{k}_{i o_{4}} \\
\mathbf{k}_{\oplus o_{1}}-\mathbf{k}_{\oplus o_{2}}+\mathbf{k}_{\oplus o_{3}}-\mathbf{k}_{\oplus o_{4}}-\mathbf{k}_{\ominus o_{1}}+\mathbf{k}_{\ominus o_{2}}-\mathbf{k}_{\ominus o_{3}}+\mathbf{k}_{\ominus o_{4}} \\
\mathbf{k}_{\mathrm{I}}-\mathbf{k}_{\mathrm{II}}+\mathbf{k}_{\mathrm{III}}-\mathbf{k}_{\mathrm{IV}}
\end{array}\right]^{(k-1)}\left[\begin{array}{c}
\delta \tilde{\mathbf{u}}_{i} \\
\delta \tilde{\mathbf{u}}_{\oplus} \\
\delta \tilde{\mathbf{u}}_{o_{1}}
\end{array}\right]^{(k)} \\
& =-\left[\begin{array}{c}
\mathbf{F}_{i} \\
\mathbf{F}_{\oplus}-\mathbf{F}_{\ominus} \\
\mathbf{F}_{o_{1}}-\mathbf{F}_{o_{2}}+\mathbf{F}_{o_{3}}-\mathbf{F}_{o_{4}}
\end{array}\right]^{(k-1)}
\end{aligned}
$$

with

$$
\begin{array}{ll}
\mathbf{k}_{\mathrm{I}}=\mathbf{k}_{O_{1} O_{1}}-\mathbf{k}_{O_{1} O_{2}}+\mathbf{k}_{O_{1} O_{3}}-\mathbf{k}_{O_{1} O_{4}}, & \mathbf{k}_{\mathrm{II}}=\mathbf{k}_{O_{2} O_{1}}-\mathbf{k}_{O_{2} O_{2}}+\mathbf{k}_{O_{2} O_{3}}-\mathbf{k}_{O_{2} O_{4}} \\
\mathbf{k}_{\mathrm{III}}=\mathbf{k}_{O_{3} O_{1}}-\mathbf{k}_{O_{3} O_{2}}+\mathbf{k}_{O_{3} O_{3}}-\mathbf{k}_{O_{3} O_{4}}, & \mathbf{k}_{\mathrm{IV}}=\mathbf{k}_{O_{4} o_{1}}-\mathbf{k}_{O_{4} O_{2}}+\mathbf{k}_{O_{4} O_{3}}-\mathbf{k}_{O_{4} O_{4}}
\end{array}
$$

\section{Emulation}

\section{III.A. The Gaussian process emulator}

Let $\eta(\cdot)$ be an expensive code, such that the associated computational cost makes it affordable to evaluate it only at a limited number of points in the input domain. This uncertainty about the output of $\eta(\cdot)$ can be modelled through a probability distribution ${ }^{26}$. Following a Bayesian treatment ${ }^{27}$, the prior beliefs about the relationship between the input and the unknown output are conditioned on a set of evaluations of $\eta(\cdot)$, therefore combining subjective and objective information. Assume that $\eta(\cdot)$ can be represented by

$$
\eta(\cdot)=\mathbf{h}(\cdot)^{\top} \boldsymbol{\beta}+Z(\cdot)
$$

where $\mathbf{h}(\cdot)$ is a vector of known functions and $\boldsymbol{\beta}$ is a vector of unknown coefficients. The vector $\mathbf{h}(\cdot)$ should be chosen to reflect the available information about the functional form of $\eta(\cdot)^{28,29}$. The function $Z(\cdot)$ is a stochastic process with mean zero and covariance function $\operatorname{Cov}(\cdot, \cdot)$. Let $\mathbf{y}=\left[\eta\left(\mathbf{x}_{\mathbf{1}}\right), \ldots, \eta\left(\mathbf{x}_{\mathbf{n}}\right)\right]^{\top}$ be a vector of observations corresponding to the inputs $\mathbf{x}_{\mathbf{1}}, \ldots, \mathbf{x}_{\mathbf{n}}$. These observations are used to update the prior distribution of $\eta(\cdot)$. An analytically convenient choice for such prior is the following Gaussian process distribution ${ }^{30}$

$$
\eta(\cdot) \mid \boldsymbol{\beta}, \sigma^{2} \sim \mathcal{N}\left(\mathbf{h}(\cdot)^{\top} \boldsymbol{\beta}, \sigma^{2} C(\cdot, \cdot)\right)
$$


where $\mathbf{h}(\cdot)$ and $\boldsymbol{\beta}$ are defined as above. The covariance function consists of a variance $\sigma^{2}$ and a correlation function $C(\cdot, \cdot)$.

Definition (Gaussian stochastic process): Let $\mathbf{x} \in \mathbb{R}^{N} \cdot Z(\cdot)$ is a Gaussian stochastic process if for any $n \geq 1$ and any choice $\left\{\mathbf{x}_{\mathbf{1}}, \ldots, \mathbf{x}_{\mathbf{n}}\right\}$, the vector $\left[Z\left(\mathbf{x}_{\mathbf{1}}\right), \ldots, Z\left(\mathbf{x}_{n}\right)\right]^{\top}$ has a multivariate normal distribution.

A popular choice of correlation function is

$$
C\left(\mathbf{x}, \mathbf{x}^{\prime}\right)=\exp \left\{-\left(\mathbf{x}-\mathbf{x}^{\prime}\right)^{\top} \mathbf{B}\left(\mathbf{x}-\mathbf{x}^{\prime}\right)\right\}
$$

where $\mathbf{B}$ is a diagonal positive definite matrix. The diagonal of $\mathbf{B}$ is a vector $\mathbf{b} \in \mathbb{R}_{+}^{n}$ of smoothness parameters. These parameters quantify the rate at which the output varies as any $\mathbf{x}$ varies. One available technique $^{31}$ to estimate these parameters is to derive the density function $f(\mathbf{B} \mid \mathbf{y})$ and obtain a maximum likelihood estimator of $\mathbf{b}$.

After conditioning on the training runs $\left(\mathbf{x}_{\mathbf{1}}, \eta\left(\mathbf{x}_{\mathbf{1}}\right)\right), \ldots,\left(\mathbf{x}_{\mathbf{n}}, \eta\left(\mathbf{x}_{\mathbf{n}}\right)\right)$ and updating the prior distribution (9), the mean of the resulting posterior distribution approximates the output of $\eta(\cdot)$ at any untried $\mathbf{x}$, whereas it reproduces the known output at each one of the inputs $\mathbf{x}_{\mathbf{1}}, \ldots, \mathbf{x}_{\mathbf{n}}$. The variance of the posterior distribution quantifies the uncertainty that arises from having only a limited number of evaluations of $\eta(\cdot)$ 32. Very conveniently, the posterior is also a Gaussian process distribution of the form

$$
\eta(\cdot) \mid \mathbf{y}, \sigma^{2} \sim \mathcal{N}\left(m^{* *}(\cdot), \sigma^{2} C^{* *}(\cdot, \cdot)\right)
$$

Since it can be shown that $m^{* *}(\cdot)$ does not include any term involving the expensive code $\eta(\cdot)$, it is a fast approximation of $\eta(\mathbf{x})$ for any $\mathbf{x}$ in its domain. The conditioning on $\sigma^{2}$ can also be eliminated, such that

$$
\frac{\eta(\mathbf{x})-m^{* *}(\mathbf{x})}{\widehat{\sigma} \sqrt{\frac{(n-q-2) C^{* *}(\mathbf{x})}{n-q}}} \sim t_{n-q}
$$

which is a Student's t-distribution with $n-q$ degrees of freedom (not to be confused with the degrees of freedom in a finite element method context). The complete process is summarised in Algorithm 1. The expressions defined above, such as $m^{* *}(\cdot)$ and $C(\cdot, \cdot)^{* *}$, are defined in the Appendix.

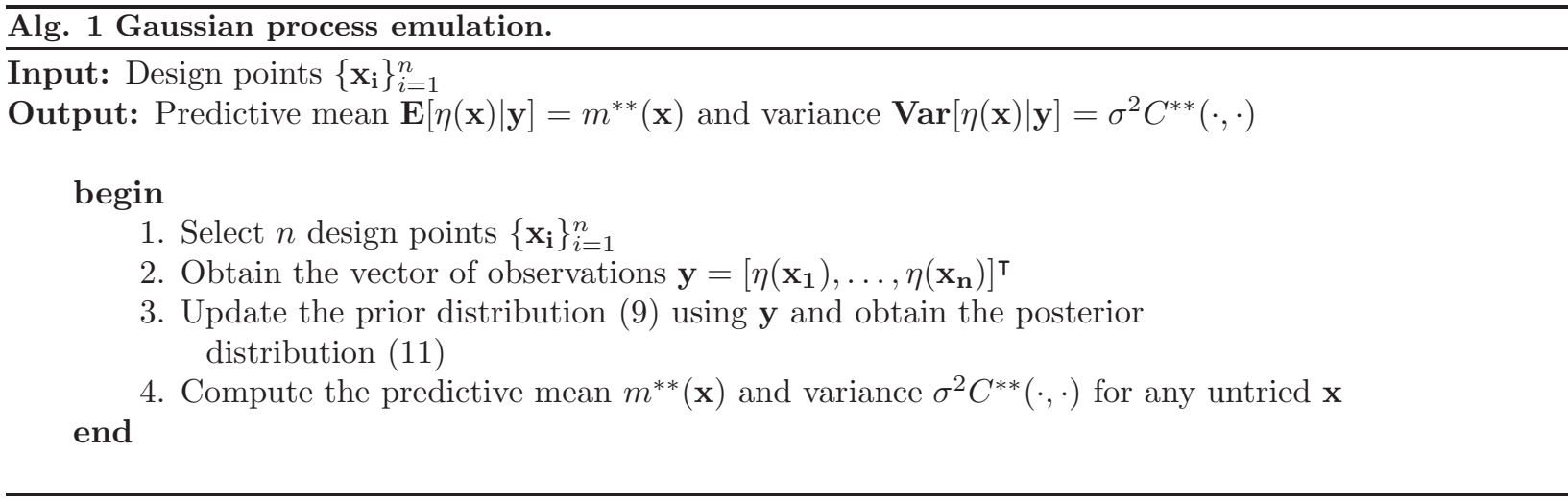

\section{III.B. Uncertainty Analysis}

Being the implementation of a computer code, a simulator $\eta(\cdot)$ is deterministic. This means that if it is run repeatedly with the same input $\mathbf{x}$, it will always return the same output $\mathbf{y}=\eta(\mathbf{x})$. However, the inputs we are interested in are physical constants, the values of which are unknown. In the probabilistic uncertainty analysis of deterministic simulators, the values of the uncertain inputs are considered to be a random variable $\mathbf{X}$. Thus, the output of the simulator is also a random variable, denoted by $\mathbf{Y}=\eta(\mathbf{X})$. To begin the uncertainty analysis, the first stage is to quantify the uncertainty in $\mathbf{X}$ by specifying a probability distribution $\mathcal{F}(\mathbf{X})$. The aim of uncertainty analysis is to propagate the uncertainty in $\mathbf{X}$ through the simulator $\eta(\cdot)$ in order to characterise the distribution of $\mathbf{Y}$. If $\eta(\cdot)$ were not an expensive simulator, the most straightforward uncertainty analysis would proceed by drawing a large sample $\left\{\mathbf{x}_{\mathbf{1}}, \ldots, \mathbf{x}_{\mathbf{N}}\right\}$ from the

6 of 14 
input distribution $\mathcal{F}(\mathbf{X})$ and then running the simulator at each of them. This would result in a sample of outputs $\left\{\mathbf{y}_{\mathbf{1}}, \ldots, \mathbf{y}_{\mathbf{N}}\right\}$ from which any statistic or summary $\mathcal{S}(\mathbf{Y})$ can be estimated. $\mathcal{S}(\mathbf{Y})$ could be the mean, the variance, a particular quantile, or any other summary.

A straightforward way to use a GPE to carry out uncertainty analysis would be to substitute $\eta(\cdot)$ with the predictive mean $m^{* *}(\mathbf{x})$. Potentially, this predictive mean can be evaluated a large number of times at any untried input at very low cost, alleviating the computational burden of the expensive simulator. However, this basic Monte Carlo approach does not incorporate the fact that any summary $\mathcal{S}(\mathbf{Y})$ is a function of an unknown quantity and thus is itself uncertain. The solution to this is detailed in Algorithm 2, which is a modified Monte Carlo algorithm proposed in ${ }^{33}$ that incorporates the uncertainty inherent to $\mathcal{S}(\mathbf{Y})$ into the analysis.

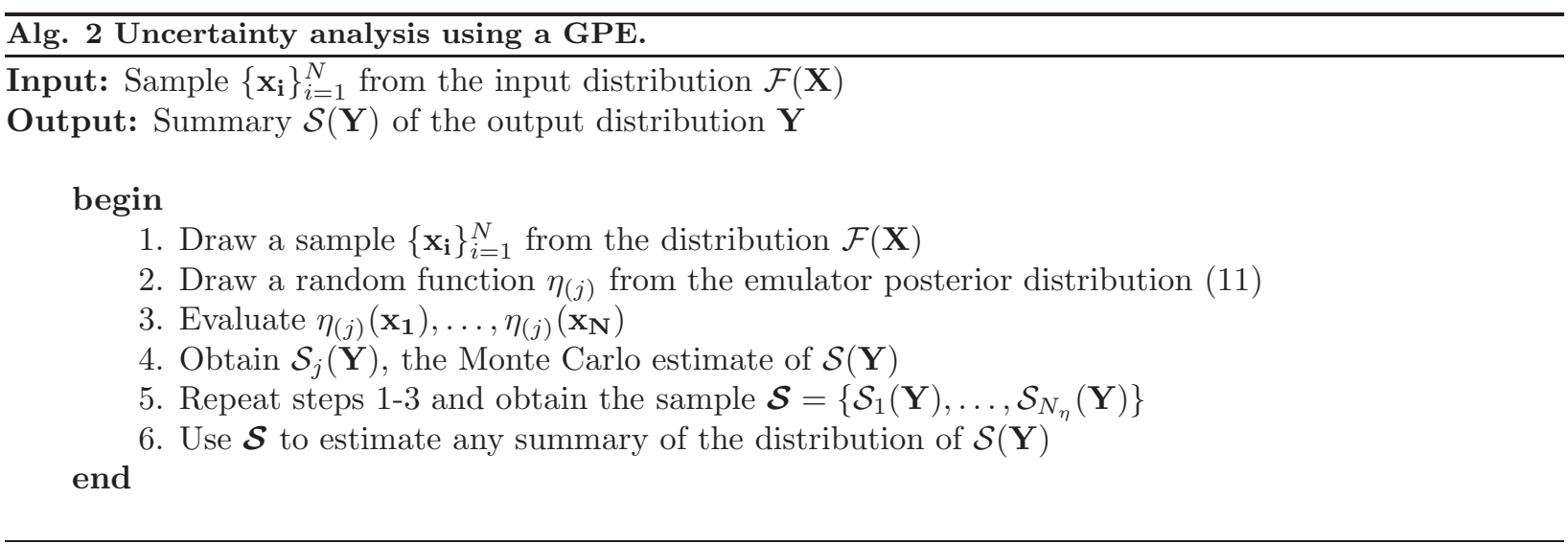

\section{Finite element modelling}

In this section, we consider the fully coupled two-scale finite element modelling of a honeycomb-cored panel under in-plane loading conditions. The particular honeycomb microstructure has been obtained from Burlayenko et al. ${ }^{11}$ and consists of a periodic arrangement of aluminum hexagonal honeycomb core filled with polyvinyl chloride (PVC) foam. The geometric parameters which define the periodic microstructure are the angle $\theta$, the in-plane rotation $\psi$, the thickness $t_{c}$ and the lengths $T$ and $R$ (refer to Figure 4 ). In this

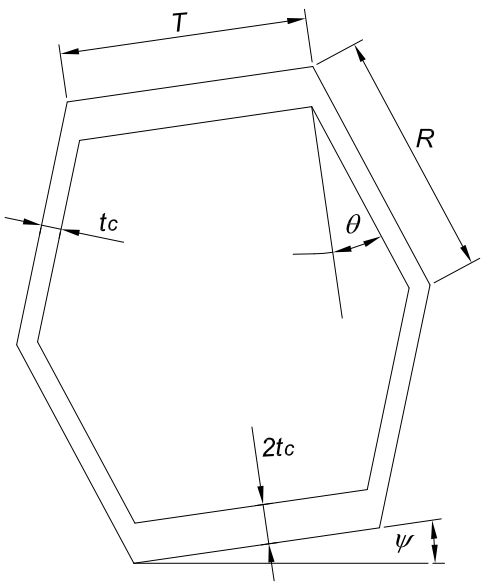

Figure 4. Geometric parameters which define the honeycomb microstructure.

study, the corresponding baseline values are taken as: $R=1 \mathrm{~mm}, T=1 \mathrm{~mm}, t c=0.08 \mathrm{~mm}, \theta=30^{\circ}$ and $\psi=0^{\circ}$.

The materials are modelled here by an elastic-perfectly plastic von Mises law. The corresponding mechanical properties for aluminum are: Young's modulus $E=72 \mathrm{GPa}$, Poisson's ratio $\nu=0.31$ and yield stress $\sigma_{y}=405 \mathrm{MPa}^{11}$. For the PVC filler, we adopt a Young's modulus $E=0.23 \mathrm{GPa}$, Poisson's ratio 
$\nu=0.32$ and yield stress $\sigma_{y}=5.75 \mathrm{MPa}^{11}$. For all of the finite element analyses we assume plane strain conditions under an infinitesimal strains regime.

To eliminate volumetric locking, the F-Bar methodology ${ }^{34}$ is adopted throughout. A typical RVE finite element mesh is shown in Figure 5, and contains 552 F-bar four-noded quadrilateral elements with a total

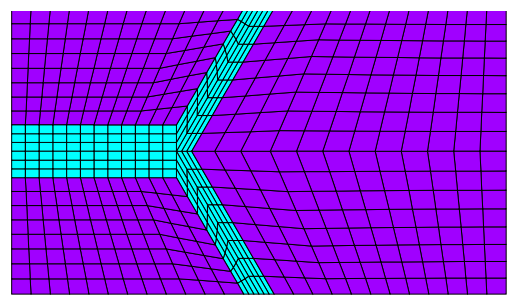

Figure 5. Typical microscopic finite element mesh adopted for the computational homogenisation of the periodic microstructure.

of 606 nodes.

The computational effort required for the solution of the (microscopic) RVE equilibrium problem is directly related to the number of degrees of freedom of the discretised RVE domain. The presence of both staggered-translational and point symmetry conditions allows the choice of an RVE domain comprising only one quarter of the original domain, in which substantial savings in terms of computing times and memory requirements are achieved.

The macroscopic problem consists of a $40 \times 40 \mathrm{~cm}$ panel whose material law is defined by the computational homogenisation of the above honeycomb microstructure. A mesh of $16 \mathrm{~F}$-bar four-noded quadrilateral elements with a total of 25 nodes is used to discretise one symmetric portion of the problem domain with appropiate boundary constraints imposed along the symmetry lines. The macroscopic finite element mesh is shown in Figure 6.

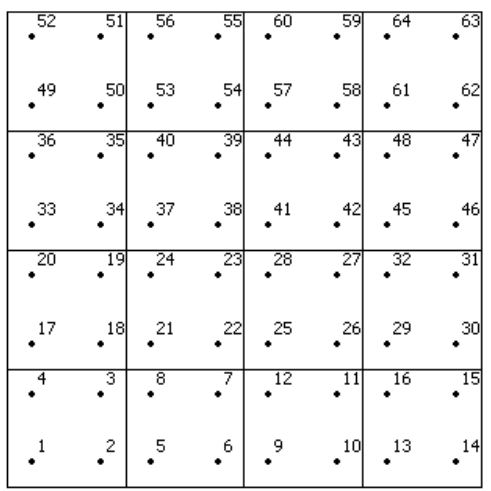

Figure 6. Typical macroscopic finite element mesh used in the modelling of the foam-filled honeycomb-cored panel under in-plane loading conditions. Gauss quadrature points are also shown here.

The loading programme consists of applying a prescribed displacement of $2 \mathrm{~cm}$ in three different directions as shown in Figure 7. In all of the cases, $x$ and $y$-axes coincide respectively with the horizontal and vertical

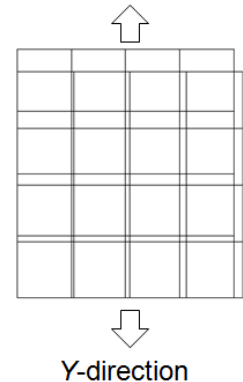

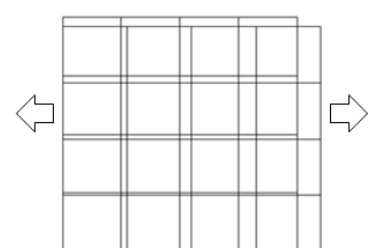

$X$-direction

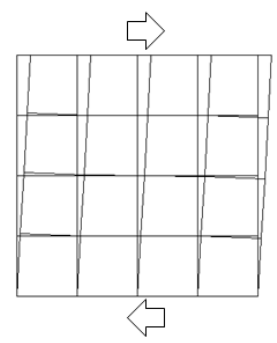

$X Y$-direction

Figure 7. Loading programmes adopted for the analysis of the mechanical response of the honeycomb-cored panel under in-plane loading conditions. 
directions. The $x y$-direction is associated with in-plane shear deformation of the panel. The total prescribed displacement is applied in each direction of analysis in 10 incremental steps. However, when no convergence is detected in the solution of the RVE equilibrium problem at any macroscopic Gauss point, smaller load increments are taken to ensure the success of the whole macroscopic loading programme. We remark that during each load step of the fully coupled two-scale analysis the macroscopic equilibrium problem is solved simultaneously with 64 RVE equilibrium problems at the macroscopic Gauss-point level (refer to Figure 6 to see the macroscopic Gauss-points).

In order to introduce uncertainty in the material definition of the above panel, we propose a modelling strategy which perturbs each of the geometric parameters shown in Figure 4. As we are interested in understanding the influence of each of these parameters on the overall mechanical response of the panel, for each analysis, a single parameter is perturbed in turn, about its baseline value at each (macroscopic) Gauss-point of the macroscopic finite element mesh. The procedure is repeated again, for a second parameter in each direction of analysis, and so on.

\section{Numerical results}

As commented in the previous section, a study of the influence of random geometric parameters on the mechanical response of the homogenised material is performed. The numerical results shown here are the effect of the incidence of the angle $\theta$, the in-plane rotation $\psi$, the thickness $t_{c}$ and the lengths $T$ and $R$, on the force-displacement curves of the honeycomb-cored panel considered in this work for the three loading directions (refer to Figure 7). The forces are determined from the sum of nodal reactions along the loading directions. In particular, we are interested in the probabilistic characterisation of the mechanical response for a range of prescribed displacements up to $2 \mathrm{~cm}$.

In order to gain insight into the deformation mechanisms, Figure 8 shows some typical results obtained from our multi-scale analyses when the baseline values of $R, T, t c, \psi$ and $\theta$ are considered in the definition of the microstructure. The contour plots for the displacement field at the macroscopic scale, and equivalent plastic strains at the microscopic scale (at Gauss-point 1 of the macroscopic mesh) at the end of the loading programme are shown here, for the three loading directions. For these three directions, it is possible to observe a large accumulation of plastic strains localised in the PVC filler, particularly in the centres of each hexagon. An interesting phenomenon is also observed in Figure 8(f), in which a large amount of plastic dissipation is also concentrated in the region next to the aluminium-PVC contact, revealing potential problems of debonding during shear deformation.

As explained in the previous section, the numerical simulations are performed by perturbing each of the geometric parameters. The assumed input distribution for each length $(R, T$, and $t c)$ was chosen to be Gaussian with a mean equal to the baseline value and with a variance such that $5 \%$ of the baseline value equals 3 standard deviations. Consequently, for each Gauss-point, $R \sim \mathcal{N}\left(1,(0.05 / 3)^{2}\right), T \sim \mathcal{N}\left(1,(0.05 / 3)^{2}\right)$, $t c \sim \mathcal{N}\left(0.08,(0.05 * 0.08 / 3)^{2}\right)$. For the case of the angles we considered a standard deviation of 1 degree. Thus, $\theta \sim \mathcal{N}(30,1), \psi \sim \mathcal{N}(0,1)$.

The statistics we are interested in determining are the 5-th, 50-th and 95-th percentiles of the force for each displacement level (assumed to be from 0 to $2 \mathrm{~cm}$ with a $0.2 \mathrm{~cm}$ increment). As mentioned before, if it were not expensive to generate every force-displacement curve for a given value of a random parameter, say $\theta$, then it would suffice to run a Monte Carlo analysis for a large number of realisations of $\theta$, and the statistics of the mechanical response could be approximated to an arbitrary degree of accuracy. Since this is not the case, a small number of realisations of the force-displacement curve were performed for a perturbation of each parameter in turn. After an inspection of these numerical results (not shown here), it is possible to observe that the parameters $\psi, t_{c}, T$ and $R$ have very little influence on the overall mechanical response of the panel, due to the proximity of the curves in each graph. However, a greater influence of the angle $\theta$ on the response of the panel was observed, particularly in the $x$ and $y$-loading directions.

Since the four parameters $\psi, t_{c}, T$ and $R$ have virtually no influence on the mechanical response, we take into consideration only the parameter $\theta$ in all of our uncertainty analyses. Here, a small set of realisations of the parameter $\theta$ is taken as the inputs upon which a GPE is built. Based on that small number of realisations, Figure 9 shows a Kernel density estimation of the reaction force (at the end of the loading programme) for the three loading directions when the parameter $\theta$ is perturbed about its baseline value.

In order to infer the percentiles, we use the estimation algorithm proposed in $^{29}$, which combines the implementations of Algorithm 1 and Algorithm 2. For this particular parameter, the emulated percentiles 


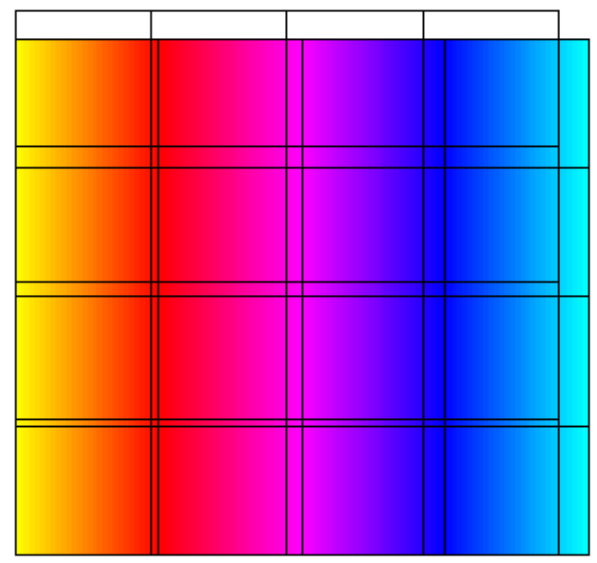

(a) Loading in the $x$-direction. Countour plot of the displacement field in the $x$-direction for the macroscopic scale.

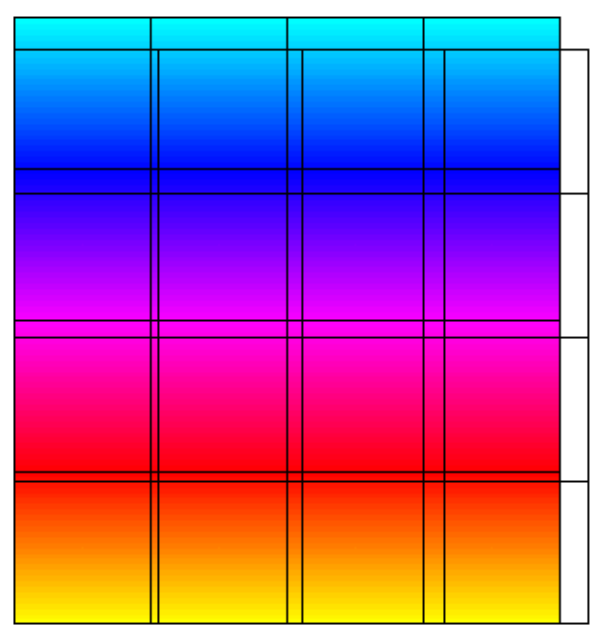

(c) Loading in the $y$-direction. Countour plot of the displacement field in the $y$-direction for the macroscopic scale.

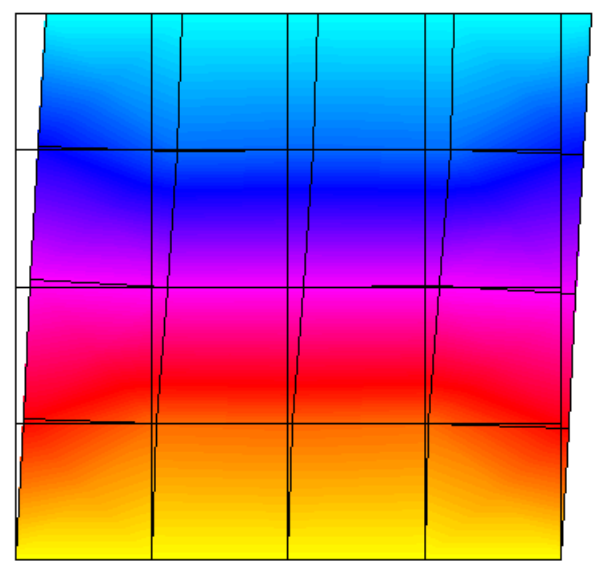

(e) Loading in the $x y$-direction. Countour plot of the displacement field in the $x$-direction for the macroscopic scale.

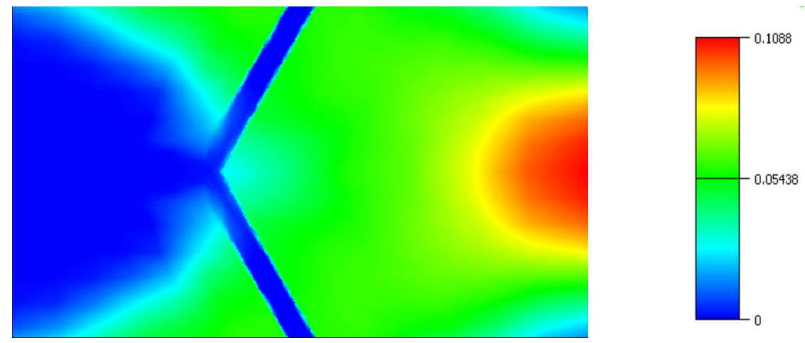

(b) Loading in the $x$-direction. Countour plot of the equivalent plastic strains at the microscopic scale.

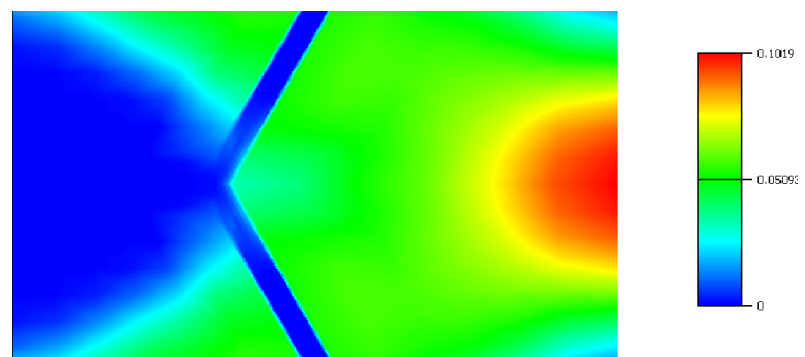

(d) Loading in the $y$-direction. Countour plot of the equivalent plastic strains at the microscopic scale.
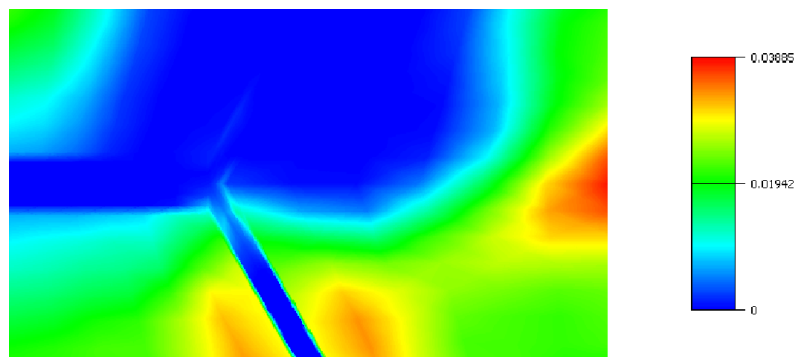

(f) Loading in the $x y$-direction. Countour plot of the equivalent plastic strains at the microscopic scale.

Figure 8. Contour diagrams for the displacement field at the macroscopic scale, and equivalent plastic strains at the microscopic scale (at Gauss-point 1 of the macroscopic mesh) at the end of the loading programme. 


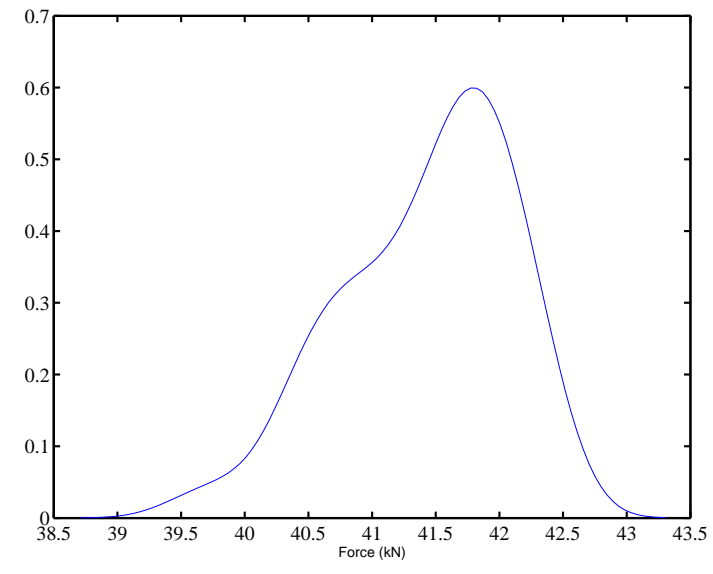

(a) Loading in the $x$-direction. Reaction force in the $x$-direction.

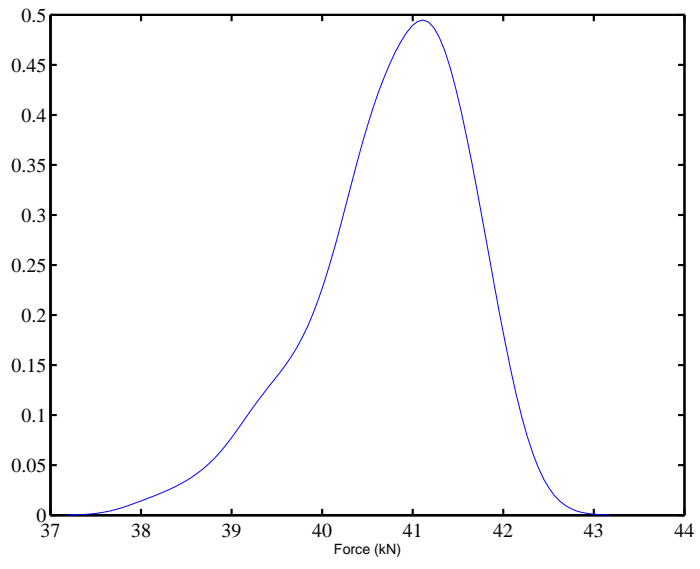

(b) Loading in the $y$-direction. Reaction force in the $y$-direction

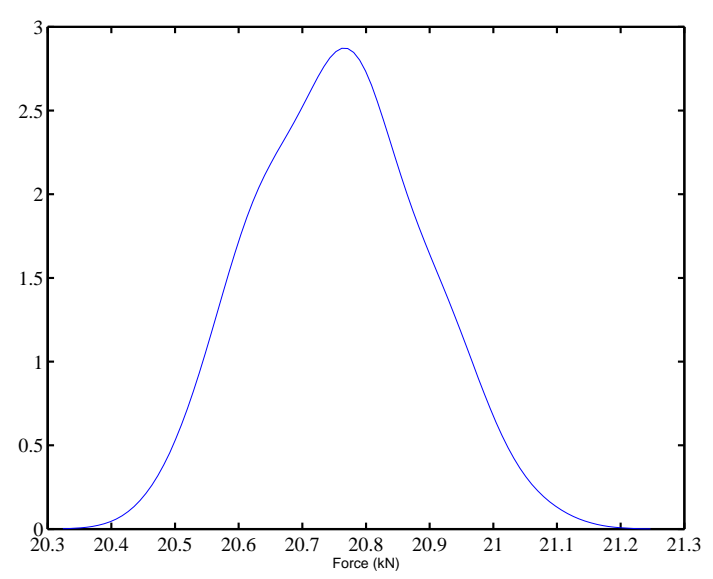

(c) Loading in the $x y$-direction. Reaction force in the $x$-direction.

Figure 9. Kernel density estimation of the reaction force for the three loading directions (at the end of the loading programme), for a small number of realisations, when the parameter $\theta$ is perturbed about its baseline value. 
of the force-displacement curves are shown in Figure 10, for each loading direction.

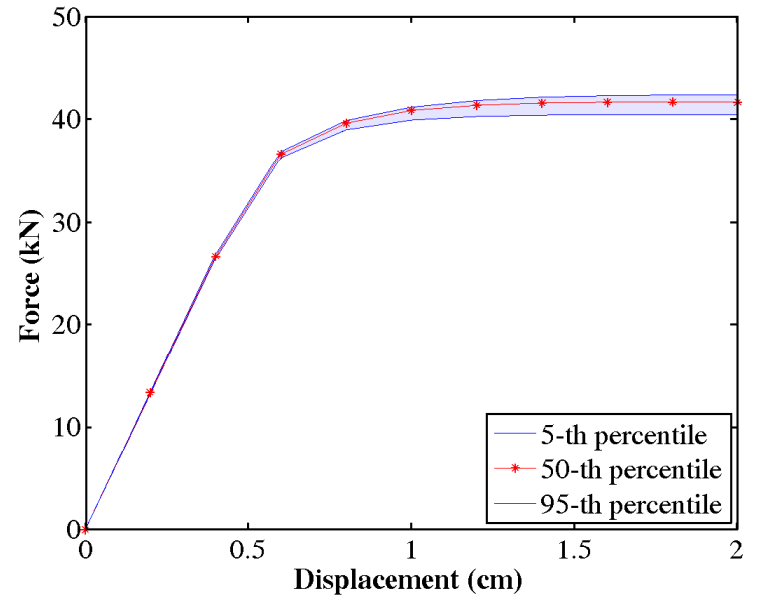

(a) Force-displacement curve in the $x$-direction.

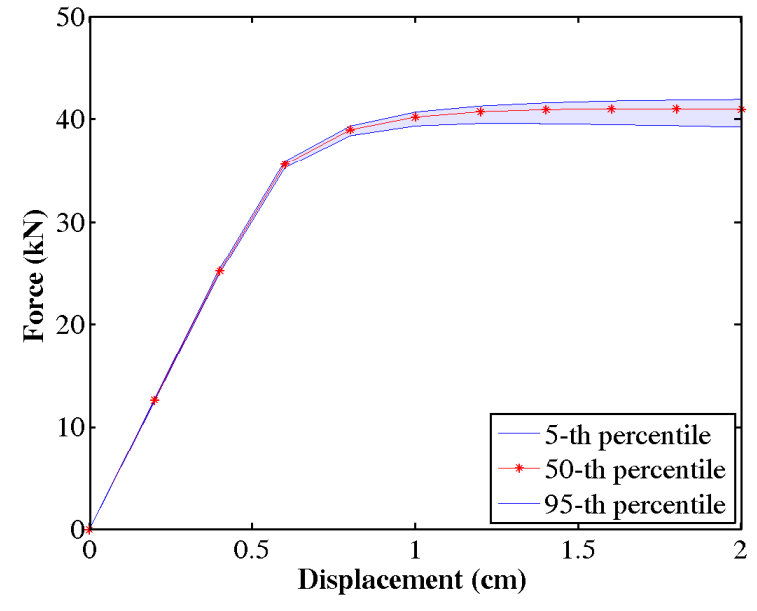

(b) Force-displacement curve in the $y$-direction.

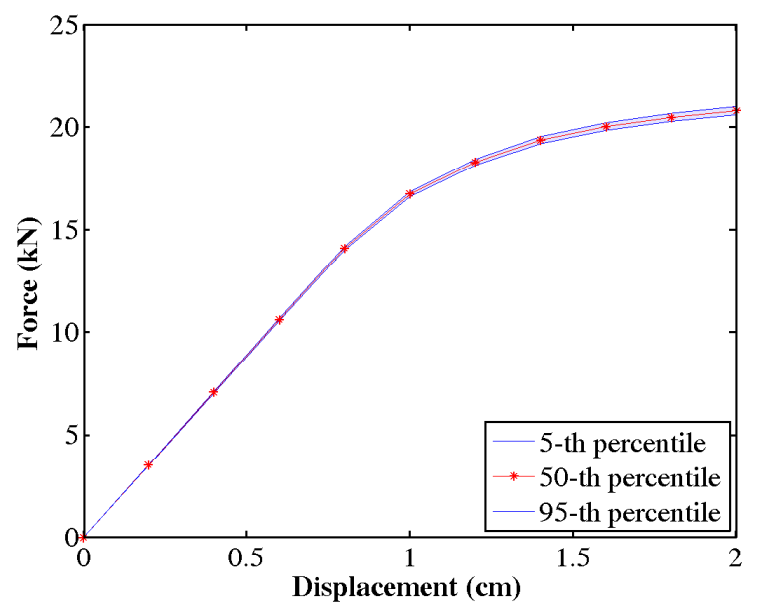

(c) Force-displacement curve in the $x y$-direction.

Figure 10. Force-displacement curves with random angle $\theta$, such that $\theta \sim \mathcal{N}(30,1)$. The emulated 5-th and 95-th percentiles are shown in the lower and upper solid lines, respectively. The median is shown as a solid dotted line.

For the $x$ and $y$-directions, we can observe a similar degree of sensitivity of the mechanical response to changes in the angle $\theta$. However, during the particular mechanism of shear deformation, the estimated mechanical response shows no significant sensitivity to changes in this parameter due to the proximity of the lower and upper curves associated to the 5-th and 95-th percentiles of the force, respectively.

\section{Concluding remarks}

This paper investigated the uncertainty in the mechanical response of foam-filled honeycomb cores by means of a finite element-based multi-scale framework. A periodic arrangement of aluminum honeycomb core filled with polyvinyl chloride (PVC) foam was chosen to study its stochastic mechanical properties and the corresponding uncertainty analyses were carried out through a metamodelling approach. Rather than carrying out a full Monte Carlo analysis, which could easily become unaffordable, GPEs were used to estimate the percentiles of the probability distribution of the load induced by random geometric properties in the microstructure. For some prescribed displacements, a reduced number of realisations of the angle $\theta$ was taken as the inputs upon which the GPEs were built. The advantage of this strategy was demonstrated by exploiting the implicit symmetry conditions existing in the RVE, along with the adoption of the metamodelling approach. That way, we were able to carry out fully coupled multi-scale analyses which would 
otherwise have been very computationally expensive.

\section{Acknowledgments}

E.I. Saavedra Flores gratefully acknowledges the support of the Department of Civil Engineering, University of Santiago, Chile.

E.I. Saavedra Flores, F.A. DiazDelaO and M.I. Friswell also acknowledge the support of the European Research Council through project 247045 entitled "Optimisation of Multi-scale Structures with Applications to Morphing Aircraft".

\section{References}

1 Miehe, C., Shotte, J., and Lambrecht, M., "Homogenization of inelastic solid materials at finite strains based on incremental minimization principles. Application to the texture analysis of polycrystals," Journal of the Mechanics and Physics of Solids, Vol. 50, No. 10, 2002, pp. 2123-2167.

2 Matsui, K., Terada, K., and Yuge, K., "Two-scale finite element analysis of heterogeneous solids with periodic microstructures," Computers and Structures, Vol. 82, 2004, pp. 593-606.

3 Pellegrino, C., Galvanetto, U., and Schrefler, B. A., "Numerical homogenization of periodic composite materials with non-linear material components," International Journal for Numerical Methods in Engineering, Vol. 46, 1999, pp. 1609-1637.

4 Castaneda, P. P., "The effective mechanical properties of nonlinear isotropic composites," Journal of the Mechanics and Physics of Solids, Vol. 39, 1991, pp. 45-71.

5 Giusti, S. M., Blanco, P. J., de Souza Neto, E. A., and Feijóo, R. A., "An assessment of the Gurson yield criterion by a computational multi-scale approach," Engineering Computations, Vol. 26, No. 3, 2009, pp. 281-301.

6 Speirs, D. C. D., de Souza Neto, E. A., and Perić, D., "An approach to the mechanical constitutive modelling of arterial tissue based on homogenization and optimization," Journal of Biomechanics, Vol. 41, 2008, pp. 2673-2680.

7 Bezazi, A., Remillat, C., Innocenti, P., and Scarpa, F., "In-plane mechanical and thermal conductivity properties of a rectangular-hexagonal honeycomb structure," Composite Structures, Vol. 84, 2008, pp. 248-255.

8 Wu, C. L., Week, C. A., and Sun, C. T., "Improving honeycomb core sandwich structures for impact resistance," Journal of Advanced Materials, 1995, pp. 41-47.

9 Vaidya, U. K., M/V Kamath, Mahfuz, H., and Jeelani, S., "Low velocity impact response of resin infusion molded foam filled honeycomb sandwich composites," Journal of Reinforced Plastic Composites, Vol. 17, No. 9, 1998, pp. 819-849.

10 Vaziri, A., Xue, Z., and Hutchinson, J. W., "Metal sandwich plates with polymer foam-filled cores." Journal of Mechanics of Materials and Structures, Vol. 1, No. 1, 2006, pp. 95-125.

11 Burlayenko, V. N. and Sadowski, T., "Effective elastic properties of foam-filled honeycomb cores of sandwich panels," Composite Structures, Vol. 92, 2010, pp. 2890-2900.

12 Saavedra Flores, E. I. and de Souza Neto, E. A., "Remarks on symmetry conditions in computational homogenisation problems," Engineering Computations, Vol. 27, No. 4, 2010, pp. 551-575.

13 Ohno, N., Matsuda, T., and Wu, X., "A homogenization theory for elastic-viscoplastic composites with point symmetry of internal distributions," International Journal of Solids and Structures, Vol. 38, 2001, pp. $2867-2878$.

14 Zhou, Z., Ong, Y., Nair, P., Keane, A., and Lum, K., "Combining Global and Local Surrogate Models to Accelerate Evolutionary Optimization," IEEE Transactions on Systems, Man and Cybernetics, Part C, Vol. 36, No. 6, 2006, pp. 814-823. 
15 Forrester, A. I. J., Sóbester, A., and Keane, A. J., Engineering Design via Surrogate Modelling. A Practical Guide, J. Wiley and Sons, Chichester, U. K., 2008.

16 Kleijnen, J. P. C., "Kriging metamodeling in simulation: a review," European Journal of Operational Research, Vol. 192, No. 3, 2009, pp. 707-716.

17 Santner, T., Williams, B., and Notz, W., The Design and Analysis of Computer Experiments, Springer Series in Statistics, London, UK, 2003.

18 Sacks, J., Welch, W., Mitchell, T., and Wynn, H., "Design and Analysis of Computer Experiments," Statistical Science, Vol. 4, No. 4, 1989, pp. 409-435.

19 Xiong, Y., Chen, W., Appley, D., and Ding, X., "A non-stationary covariance-based Kriging method for metamodelling in engineering design," International Journal for Numerical Methods in Engineering, Vol. 71, 2006, pp. $733-756$.

20 DiazDelaO, F. A. and Adhikari, S., "Structural dynamic analysis using Gaussian process emulators," Engineering Computations, Vol. 27, No. 5, 2010, pp. 580 - 605.

21 DiazDelaO, F. A. and Adhikari, S., "Gaussian process emulators for the stochastic finite element method," International Journal for Numerical Methods in Engineering, Vol. 87, No. 6, 2011, pp. 521-540.

22 Hill, R., "A self-consistent mechanics of composite materials," Journal of the Mechanics and Physics of Solids, Vol. 13, No. 4, 1965, pp. 213-222.

23 Mandel, J., Plasticite Classique et Viscoplasticite. CISM Lecture Notes, Springer-Verlag, Udine, Italy, 1971.

24 de Souza Neto, E. A., Perić, D., and Owen, D. R. J., Computational Methods for Plasticity: Theory and Applications, Wiley: Chichester, 2008.

25 Lemaitre, J. and Chaboche, J. L., Mechanics of Solid Materials, Cambridge University Press, Cambrige, 1990.

26 Oakley, J., "Eliciting Gaussian Process Priors for Complex Computer Codes," The Statistician, Vol. 51, No. 1, 2002, pp. 81-97.

27 O'Hagan, A., "Bayesian analysis of computer code outputs: a tutorial," Reliability Engineering \& System Safety, Vol. 91, No. 10-11, 2006, pp. 1290-1300.

28 Oakley, J. E. and O'Hagan, A., "Probabilistic Sensitivity Analysis of Complex Models: a Bayesian Approach," Journal of the Royal Statistical Society B, Vol. 66, No. 3, 2004, pp. 751-769.

29 Oakley, J., "Estimating Percentiles of Computer Code Outputs," Applied Statistics, Vol. 53, 2004, pp. 8393.

30 Haylock, R. and O'Hagan, A., Bayesian Statistics 5, chap. On inference for outputs of computationally expensive algorithms with uncertainty on the inputs, Oxford University Press, Oxford, UK, 1996.

31 Haylock, R., Bayesian Inference About Outputs of Computationally Expensive Algorithms with Uncertainty on the Inputs, Ph.D. thesis, University of Nottingham, Nottingham, UK, 1996.

32 Rougier, J., "Probabilistic inference for future climate using an ensemble of climate model evaluations," Climatic Change, Vol. 81, No. 3, 2007, pp. 247-264.

33 Oakley, J. E. and O'Hagan, A., "Bayesian inference for the uncertainty distribution of computer model outputs," Biometrika, Vol. 89, 2002, pp. 769-784.

34 de Souza Neto, E. A., Perić, D., Dutko, M., and Owen, D. R. J., "Design of simple low order finite elements for large strain analysis of nearly incompressible solids," International Journal of Solids and Structures, Vol. 33, 1996, pp. 3277-3296. 